\title{
Lidil
}

Revue de linguistique et de didactique des langues

49 | 2014

L'analyse des données didactiques

\section{L'analyse des pratiques en classe de français : théories et méthodes}

\section{Roxane Gagnon et Sandra Canelas-Trevisi}

\section{(2) OpenEdition}

1 Journals

Édition électronique

URL : http://journals.openedition.org/lidil/3417

DOI : 10.4000/lidil.3417

ISSN : 1960-6052

Éditeur

UGA Éditions/Université Grenoble Alpes

\section{Édition imprimée}

Date de publication : 25 mai 2014

Pagination : 5-15

ISBN : 978-2-84310-272-1

ISSN : $1146-6480$

\section{Référence électronique}

Roxane Gagnon et Sandra Canelas-Trevisi, «L'analyse des pratiques en classe de français : théories et méthodes », Lidil [En ligne], 49 | 2014, mis en ligne le 25 novembre 2015, consulté le 22 septembre 2020. URL : http://journals.openedition.org/lidil/3417 ; DOI : https://doi.org/10.4000/lidil.3417 


\title{
L'analyse des pratiques en classe de français : théories et méthodes
}

\author{
Roxane Gagnon* et Sandra Canelas-Trevisi**
}

Les questions relatives à l'enseignement et à l'apprentissage institutionnels du français ont toujours pris en compte ce qui se passe dans les pratiques en classe. Dans les travaux de recherche ainsi que dans les textes émanant d'institutions sociopolitiques diverses, les pratiques sont tantôt questionnées de manière explicite, tantôt placées en toile de fond. Ainsi, que ce soit en français langue première, en français langue seconde et étrangère ${ }^{1}$, nous considérons que le mode de traitement des pratiques permet de distinguer deux grands ensembles de travaux (Bayer \& Ducrey, 2001). Le premier réunit les questions ayant trait à l'amélioration de l'enseignement selon un projet culturel et social historiquement situé : les pratiques ne sont pas explicitement analysées, mais elles sont convoquées en tant que révélateur d'une crise à résoudre, le plus souvent, par la réorganisation des contenus enseignés. Les travaux qui s'intéressent explicitement aux pratiques en classe appartiennent plutôt au second ensemble. Comme le souligne Brousseau (1998), ils proposent des analyses, plus ou moins strictement délimitées, d'une des composantes des pratiques : l'enseignant et ses conduites, l'élève et ses difficultés, le degré d'adéquation des contenus et des supports de l'enseignement aux attentes sociales.

Organisés à partir de questions émanant d'instances scientifiques et sociales multiples, ces textes laissent voir de manière inégale les relations entre les acteurs engagés dans les pratiques, les actions et les discours qui les définissent. Or nous considérons qu'il est utile «de [nous] demander quel type de rapport entretiennent les modèles théoriques

* GRAFE, Université de Genève-FPSE.

** LIDILEM, Université Grenoble Alpes et GRAFE, Université de Genève FPSE.

1. Étiquettes largement discutées mais que nous utilisons ici par commodité. 
construits pour rendre compte d'une pratique sociale comme celle de l'enseignant face à ses élèves, avec la réalité de cette pratique et les véritables enjeux de son fonctionnement» (Nonnon, 1990, p. 155).

Comme les didactiques des disciplines, la didactique du français est aujourd'hui engagée dans la constitution d'un champ susceptible de fédérer ces questions de recherche, même si des divergences existent :

On peut distinguer aujourd'hui dans ce mouvement qui tend vers la constitution d'une science, ou, disons plus modestement, d'un champ disciplinaire «didactique», plusieurs démarches qui, certes, ne s'excluent pas mutuellement, mais à l'intérieur desquelles — et c'est normal — on peut aussi déceler des luttes de territoire (Dorier, Leutenegger \& Schneuwly, 2013, p. 16).

La didactique du français est le produit d'un processus de disciplinarisation datant d'un petit demi-siècle. Elle se définit par «un corps de concepts originaux, relativement stables, avec des démarches de recherche systématiques, notamment empiriques » (Dorier, Leutenegger \& Schneuwly, 2013, p. 10) et par la constitution d'un appareil institutionnel porté par une communauté de chercheurs. Soulignons également qu'elle s'est développée à partir d'un espace professionnel préexistant (celui des métiers de l'enseignement), à l'instar, entre autres, de la médecine et des sciences de l'éducation. Cela en accentue probablement l'hétérogénéité, par rapport aux disciplines se développant selon une logique interne forte, dont les apports sont investis secondement dans la professionnalisation.

Par ailleurs, s'il parait incontestable que les pratiques d'enseignement et d'apprentissage institutionnels, d'une part, les pratiques de formation professionnelle des enseignants, d'autre part, font l'objet de questions de recherche en didactique du français, il est tout aussi certain que des recherches, diverses sur le plan théorique et méthodologique, contribuent à leur théorisation. Les données empiriques recueillies dans les situations de formation constituent en effet un champ d'investigation que la didactique du français et les didactiques des disciplines partagent notamment avec la psychologie cognitive, la sociologie du travail et le vaste champ des recherches sur l'interaction.

De nombreux travaux ont été réalisés, émanant d'espaces théoriques parfois éloignés : les méthodes ethnographiques d'analyse des interactions (Filliettaz, 2008; Filliettaz \& Schubauer Leoni, 2008 ; Filliettaz, 2009); l'analyse de l'activité fondée sur la verbalisation à postériori selon les principes de l'ergonomie (Clot, 2001); l'analyse des interactions et de l'agir professoral (Cicurel, 2011); l'analyse des genres de 
discours et des activités de travail (Boutet, 2005; Faïta, 2003); l'étude de la construction située des compétences (Pekarek Doehler, 2004).

Les recherches se revendiquant explicitement de la didactique s'appuient sur un corps de concepts partagés : l'étude des objets institutionnels enseignés en classe (Schneuwly \& Dolz, 2009; Canelas-Trevisi, 2009); l'analyse des gestes professionnels des enseignants (Jorro, 2004; Jaubert \& Rebière, 2005; Garcia-Debanc, 2007; Sensevy \& Mercier, 2007); l'analyse du discours sur les savoirs professionnels (Vanhulle, 2009); l'ingénierie de formation (Chemla, 2001; Ronveaux \& Cordonier, 2007; Gagnon, 2010); la constitution des disciplines scolaires et de la forme scolaire (Reuter, 2007; Thévenaz-Christen, 2005; Vincent, 1994). Malgré leur diversité sur le plan théorique et méthodologique, ces travaux contribuent néanmoins tous à instituer les pratiques en classe en objet de recherche, en particulier par la mise en évidence de régularités.

Le domaine de recherche qui se précise aujourd'hui intègre à l'analyse des pratiques de formation le point de vue des conduites socialement attendues, telles qu'elles sont décrites dans les discours des institutions d'enseignement (le CECR pour le français langue seconde et étrangère, les programmes institutionnels des différents pays francophones pour le français langue première) et évaluées selon des critères institutionnels (DELF, DALF). L'analyse des phénomènes interactionnels fait apparaitre les procédures par lesquelles les interactants négocient la forme et le sens de leurs conduites, mobilisant des objets institutionnels sélectionnés pour être acquis / appris $^{2}$ et évalués. Ainsi, ce qui se dit et se fait dans les pratiques de formation est inscrit dans des réseaux contextuels allant des choix institutionnels des contenus et des objectifs à une reconnaissance sociale de leur degré de maitrise, mesuré par les évaluations, moment de visibilité sociale maximale.

\section{Le double objectif du numéro}

Dans la recherche didactique, la reconstruction théorique des données mobilise aujourd'hui un ensemble de concepts : transposition didactique, contrat didactique, gestes professionnels, transaction, agir enseignant, etc. Parallèlement, elle s'appuie sur des savoirs multiples issus

2. Suivant notamment Véronique (2009), nous retenons le principe de la continuité entre acquisition et apprentissage. 
d'espaces théoriques où les conduites attendues des apprenants et des enseignants sont décrites, expliquées, racontées, préfigurées selon des points de vue divers. Les frontières entre ces différents points de vue sont souvent poreuses. L'objectif de ce numéro est de cerner comment ces questions sont abordées dans le «laboratoire» du chercheur, de faire apparaitre les axes dominants ainsi que les spécificités didactiques. Partant du principe que les facteurs contextuels influencent de façon prépondérante les pratiques de formation, nous considérons que la définition de l'objet de recherche requiert un traitement théorique explicité du contexte des pratiques analysées. Cela constitue notre première ligne directrice.

Parallèlement, en écho aux références théoriques, nous voulons poursuivre la réflexion méthodologique entreprise autour de l'analyse des pratiques de formation. En effet, comme le souligne Daunay, le traitement méthodologique des interactions dans un contexte institutionnel de formation constitue un enjeu didactique déterminant. Dans la démarche de recherche en didactique, les trois pôles du triangle didactique sont envisagés dans leur interaction, même si la mise en œuvre méthodologique isole l'un des trois éléments (Daunay, 2007). Ainsi, les principes méthodologiques qui sous-tendent les procédures de recueil des données, le statut des données audio et vidéo, les outils d'analyse constituent notre seconde ligne directrice. Organisé à partir de ces deux axes, ce numéro 49 de Lidil réunit des contributions qui mettent en résonance, dans le champ de la didactique du français, les ancrages théoriques et les méthodes de recherche, dans le but de faire apparaitre des dominantes ainsi que des spécificités.

\section{Comment les contributions déclinent-elles les deux lignes directrices de la revue? L'ancrage contextuel des pratiques analysées}

Le présent numéro s'intéresse à l'analyse des pratiques institutionnelles de formation en français langue première, seconde, étrangère. Ces trois étiquettes, bien que peu consensuelles, sont utilisées ici pour exprimer notre intention d'élargir l'éventail des contextes institutionnels. Les données des contributions sont issues, tour à tour, de la classe du primaire (cycle 3 en français ou $2^{\mathrm{e}}$ cycle suisse-romand), de la classe de français au secondaire québécois et suisse-romand et de la classe de français destinée à un public non francophone d'horizons divers (demandeurs d'emploi, universitaires, professionnels). 
Dans les différentes contributions, le contexte

- fournit les clés de compréhension de procédures cognitives mises en œuvre par les élèves au moment d'apprendre la morphographie;

- est un ingrédient même du système complexe de relations et de médiations qu'implique l'enseignement de la littérature;

- éclaire l'analyse de la parole et des gestes de l'enseignant;

- oriente le déploiement de l'objet au moment d'enseigner la grammaire au secondaire;

- surdétermine les dimensions prises en considération dans le discours.

Les traitements du contexte permettent d'expliciter les principes théoriques, les méthodes et les résultats des recherches.

\section{Les méthodes}

Des focalisations multiples nous aident à cerner les rapports entre les trois pôles du triangle didactique. L'agir de l'enseignant est saisi à travers des angles d'analyse divers : discursif, multimodal, ethnographique. La confrontation des pratiques effectives et déclarées laisse entrevoir les conceptions et les représentations des enseignants. L'agir des élèves est saisi à travers la construction tantôt d'un objet circonscrit, comme l'accord orthographique, tantôt d'un macro-objet, aux contours plus poreux, tel que le rapport au texte littéraire. Les dispositifs de recueil des données sont majoritairement écologiques. Quand ils convoquent une démarche expérimentale, ils sont construits à partir d'outils (texte réactif, procédures d'analyse) dont il s'agit d'observer les effets.

\section{Présentation des contributions}

Dans toutes les contributions, les théories de référence, très diversifiées, sont reconfigurées en tenant compte des relations entre les trois pôles du triangle didactique, inhérentes à la question des pratiques en classe. En parallèle sont étayés les choix méthodologiques : des dispositifs novateurs sont illustrés et certaines des contributions sont directement construites à partir des questions méthodologiques.

L'article de Brahim Azaoui est centré sur l'aspect mimo-gestuel de l'agir enseignant et porte sur l'analyse d'un corpus filmique de cours 
d'une même enseignante dans deux contextes différents : la classe de français langue seconde (élèves non francophones nouvellement arrivés en France) et celle de français langue première. Le dispositif méthodologique permet une analyse très fine faisant apparaitre, dans les deux contextes, des stratégies analogues comme le «relai» regard-geste qui permet à l'enseignante d'évaluer tout en faisant avancer le cours. À l'aide de quatre clichés, l'auteur montre un des nombreux moments où le regard, le verbal et la gestuelle contribuent à désigner le coénonciateur principal et à maintenir l'attention de la classe. Face à la complexité de la segmentation des gestes, l'auteur étaye son questionnement méthodologique et s'interroge sur les critères à adopter.

La contribution de Chiara Bemporad étudie les conduites de deux étudiantes canadiennes anglophones lors d'un exposé oral réalisé dans un cours de littérature française. Trois sortes de données sont mises en relation : documents écrits nécessaires à la préparation de l'exposé et consigne; enregistrement en classe de l'exposé oral; entretien avec les deux étudiantes après l'exposé. Partant d'une conception du développement des compétences socialement, historiquement et institutionnellement situé, l'auteure décrit les opérations de médiation formative par lesquelles les deux étudiantes intègrent de nouvelles connaissances linguistiques, culturelles et disciplinaires. L'analyse de trois segments de l'exposé oral identifiés comme séquences potentiellement d'apprentissage fait apparaitre les trois types de médiation (individuelle, collaborative et collaborative avec experts) qui les caractérisent.

La recherche exposée dans la contribution d'Ecaterina Bulea et de Sylvie Jusseaume se situe dans le contexte institutionnel du FLE. Les analyses portent sur un corpus d'entretiens avec des enseignants de FLE, constitué de huit entretiens semi-directifs et de la partie «entretien» de l'instruction au sosie. Ces entretiens sont analysés en tant que textes. Selon les principes théoriques de l'interactionnisme sociodiscursif, les analyses mettent en évidence dans les textes les traces du contexte énonciatif de production, d'une part, de l'organisation textuelle d'autre part, notamment l'émergence des types de discours. Dans les entretiens d'instruction au sosie, les auteures s'intéressent en particulier au segment textuel dans lequel l'enseignant interprète son agir lors de l'opérationnalisation d'un objet particulier, à savoir le passé composé. Les analyses mettent en relation les «figures d'action» émergeant des entretiens avec la construction discursive des gestes didactiques et des contenus enseignés.

Le contexte du secondaire québécois est analysé de manière détaillée dans la contribution de Judith Émery-Bruneau. L'objectif est de 
décrire et comprendre ce qui s'y enseigne sous l'appellation «littérature». Pour y parvenir, l'auteure commence par poser au départ la littérature comme objet enseigné, qu'elle étudie ensuite en tenant compte du rapport de l'enseignant à l'objet de sa pratique. La notion de rapport à la littérature désigne les liens entre les conceptions qu'ont les enseignants de la littérature et leurs pratiques déclarées. Les données analysées comportent des entretiens semi-dirigés auprès de 10 enseignants. L'article présente les analyses des conceptions et des pratiques déclarées de ces enseignants. Cinq aspects dominants de leur rapport à la littérature sont décrits plus finement : leurs conceptions de la littérature, leurs critères de littérarité, les œuvres enseignées, les finalités de l'enseignement de la littérature et le rôle de la littérature dans le développement des savoirs. Dans la majorité des cas, le rapport des enseignants à la littérature est influencé par leurs pratiques personnelles de la littérature, leur formation initiale et surtout le contexte d'enseignement.

Le questionnement de Thierry Geoffre, solidement ancré dans le contexte institutionnel de l'école primaire en France, porte sur l'acquisition de la morphologie par les élèves de cycle 3 (âgés de 11 ans, en dernière année de scolarité primaire). Tout en mettant en discussion la possibilité de régler l'apprentissage de l'orthographe à l'école primaire, point de vue largement débattu à l'heure actuelle, l'auteur défend le principe de doter les élèves en fin de scolarité primaire d'outils d'analyse de la morphographie, notamment la chaine des accords à l'intérieur du syntagme nominal et entre le syntagme nominal sujet et le prédicat. Dans une classe de cycle 3 de l'école primaire (classe à trois niveaux, élèves âgés de 8 à 11 ans), l'auteur met en œuvre deux outils issus de l'ingénierie didactique dans deux séquences expérimentales et étudie ensuite les traces écrites de trois tests. L'objectif est de discuter la pertinence de chacun des outils en analysant le degré d'utilisation, le mode d'utilisation et enfin le décalage entre l'outil enseigné et l'outil appris. Les principes de la transposition didactique sont mis en relation avec les études sur l'acquisition de la morphographie.

Le contexte de l'école primaire en France est également au cœur de l'article de Malika Kaheraoui et Muriel Coret. La contribution s'inscrit dans un projet de recherche interdisciplinaire (mathématiques, sciences et français). Le but de la mise en dialogue des didactiques des trois disciplines est de mieux cerner les modèles didactiques utilisés en classe de français langue première, plus spécifiquement pour l'enseignement et l'apprentissage de la grammaire. Le concept d'institutionnalisation, théorisé d'abord en didactique des mathématiques, est problématisé et réinvesti pour l'analyse des premières données recueillies, encore 
très partielles. Il s'agit de séances filmées dans trois classes de cycle 3 (élèves de 9,10 ans). Une liste de marqueurs d'institutionnalisation est présentée et discutée. À partir de ce premier recueil, une réflexion est amorcée sur les formes d'institutionnalisation adoptées par la même enseignante dans chacune des trois disciplines. La comparaison avec les deux autres disciplines montre que les savoirs enseignés en grammaire sont nettement moins problématisés et que les procédures de découvertes sont plus rares.

L'identification d'un problème assez largement discuté dans le contexte du secondaire québécois est un facteur déterminant pour la recherche de Marie-Andrée Lord. C'est en effet le constat de nombreuses lacunes en orthographe et en syntaxe relevées chez les élèves au terme de la scolarité obligatoire qui a amené la chercheuse à étudier les pratiques d'enseignement de la grammaire au secondaire. L'article comporte une présentation très détaillée du recueil et du traitement des données ainsi que l'analyse critique de l'outillage méthodologique employé. L'auteure souligne en particulier que l'utilisation du synopsis pour décrire les pratiques observées amène à mieux comprendre ce qui se passe dans la classe. Pour sa valeur euristique, l'opération longue et complexe de mise à plat et de synthèse des données observées dans une classe donnée, nécessaire pour établir le synopsis, fait de l'observateur «un expert» de la classe étudiée.

La recherche de Chloé Gabathuler et de Bernard Schneuwly développe la question de la relation au texte littéraire dans les pratiques d'enseignement et d'apprentissage. Les données analysées sont issues du corpus d'une recherche sur la lecture de textes littéraires, menée dans des écoles genevoises du primaire, du secondaire I (élèves de 12 à 15 ans) et du secondaire II (élèves de 15 à 18 ans). Deux textes contrastés ont été soumis aux élèves : l'un didactisé depuis fort longtemps et l'autre, contemporain, n'ayant fait l'objet d'aucune didactisation. Pour analyser les différents types de relation au texte construits dans les classes, les auteurs ont choisi comme unité d'analyse le «jugement». La trace du jugement est repérée dans les transcriptions des situations de classe filmées. Les auteurs retiennent les segments comportant au moins une caractéristique du texte objet d'étude dans laquelle apparait un point de vue esthétique, éthique ou émotionnel. Ces catégories d'analyse sont définies dans un cadre théorique détaillé. Les résultats des analyses témoignent de la prégnance du jugement dans la relation au texte et donnent à voir l'évolution du point de vue au cours de la scolarité. 


\section{RÉFÉRENCES BIBLIOGRAPHIQUES}

BAyer, Édouard \& Ducrey, François. (2001). Une éventuelle science de l'enseignement aurait-elle sa place en sciences de l'éducation? Dans R. Hofstetter \& B. Schneuwly, Le pari des sciences de l'éducation (p. 243-277). Bruxelles : De Boeck.

Boutet, Josiane. (2005). Genres de discours et activités de travail. Dans L. Filliettaz \& J.-P. Bronckart (dir.), L'analyse des actions et des discours en situation de travail. Louvain-la-Neuve : Peters.

Brousseau, Guy. 1998. Théorie des situations didactiques. Grenoble : La pensée sauvage.

BRONCKART, Jean-Paul. (2004a). La transposition didactique dans les interventions formatives. Dans A. Faundez \& E. Mugrabi, Ruptures et continuités en éducation : aspects théoriques et pratiques (p. 79-109). Genève : Institut pour le développement et l'éducation des adultes.

BRONCKART, Jean-Paul. (2004b). Pourquoi et comment analyser l'agir verbal et non verbal en situation de travail? Dans J.-P. Bronckart \& groupe LAF (dir.), Agir et discours et situation de travail (p. 11-14). Genève : Université de Genève (Cahiers de la section des sciences de l'éducation, 103).

BRONCKART, Jean-Paul. (2008). Du texte à la langue, et retour : notes pour une reconfiguration de la didactique du français. Pratiques, 137-138, 97-116.

Canelas-Trevisi, Sandra. (2010). Les objets grammaticaux et les pratiques de classe en FLE. Dans O. Galatanu, M. Pierrard, D. Van Raemdonck, M.-E. Damar, N. Kemps \& E. Schoonheere (dir.), Enseigner les structures langagières en FLE (p. 193-202). Bruxelles : Peter Lang.

Chemla, Marie-Thérèse. (2001/2002). Formation d'enseignants : Quelles modalités pour travailler l'oral en situation d'enseignement? Repères, 24(2), 257-274.

Cicurel, Francine. (2011). De l'analyse des interactions en classe de langue à l'agir professoral : une recherche entre linguistique interactionnelle, didactique et théories de l'action, Pratiques, 149-150, 41-55.

Clot, Yves. (2001). Clinique du travail et action sur soi. Dans J.-M. Baudoin \& J. Friedrich (dir.), Théories de l'action et éducation (p. 255-277). Bruxelles : De Boeck.

DaunaY, Bertrand. (2007). État des recherches en didactique de la littérature. Revue française de pédagogie, 159, 139-189.

Dorier, Jean-Luc, Leutenegger, Francia \& Schneuwly, Bernard. (2013). Didactique en construction, constructions des didactiques. Bruxelles : De Boeck. 
FAïTA, Daniel. (2003). Apport des sciences du travail à l'analyse des activités enseignantes. Skholê, hors-série 1, 17-23.

Filliettaz, Laurent \& Schubauer Leoni, Maria Luisa. (2008). Processus interactionnels et situations éducatives. Bruxelles : De Boeck.

FiLlietTAZ, Laurent. (2009). Les formes de didactisation des instruments de travail en formation professionnelle initiale : une approche comparatiste. Travail et Apprentissages, 4, 26-56.

Gagnon, Roxane. (2010). Former à enseigner l'argumentation orale. De l'objet de formation à l'objet enseigné en classe de culture générale (Thèse de doctorat en sciences de l'éducation, Université de Genève).

Garcia-Debanc, Claudine. (2007). La reformulation orale : un élément de l'expertise professionnelle. Dans L. Talbot \& M. Bru, Des compétences pour enseigner (p. 151-168). Rennes : Presses universitaires de Rennes.

JAUBert, Martine \& ReBIÈRE, Maryse. (2005). Gestes et genres professionnels. La Lettre de l'Association, AIRDF, 36, 23-24.

Jorro, Anne. (2004). Le corps parlant de l'enseignant. Document présenté au $9^{e}$ colloque de l'AIRDF «Le français, discipline singulière, plurielle ou transversale?», Université Laval, Québec. Disponible en ligne sur $<$ www.colloqueairdf.fse.ulaval.ca/fichier/Symposium_Bucheton/Jorro. pdf $>$.

Matthey, Marinette \& Simon, Diana Lee (dir.). (2009). Altérité et formation des enseignants, Lidil, 39.

Nonnon, Élisabeth. (1990). Est-ce qu'on apprend en discutant? Interaction maitre-élèves en S.E.S. Dans F. François (dir.), La communication inégale. Heurs et malheurs de l'interaction verbale (p. 147-212). Neuchâtel : Delachaux \& Niestlé.

Pekarek Doehler, Simona. (2004). Grammaire et interaction sociale : les processus référentiels dans la conversation. Manuscrit (thèse d'habilitation, Université de Bâle).

RABATEL, Alain \& Blanc, Nathalie (dir.). (2011). Le rapport au savoir dans les discours professionnels, Lidil, 43.

ReUter, Yves. (2007). La conscience disciplinaire. Éducation et didactique, 1(2), 57-70.

Ronveaux, Christophe \& Cordonier, Noël. (2007). L'alternance au service des objets de savoirs disciplinaires, de l'objet de formation à l'objet d'enseignement. Dans F. Merhan, C. Ronveaux \& S. Vanhulle, Alternances en formation (p. 143-163). Bruxelles : De Boeck.

Sensevy, Gérard \& Mercier, Alain. (2007). Agir ensemble. L'action didactique conjointe du professeur et des élèves. Rennes : Presses universitaires de Rennes.

Schneuwly, Bernard \& Dolz, Joaquim (dir.). (2009). Des objets enseignés en classe de français. Le travail de l'enseignant sur la rédaction de 
textes argumentatifs et sur la subordonnée relative. Rennes: Presses universitaires de Rennes.

Thévenaz-Christen, Thérèse. (2005). Les prémices de la forme scolaire. Étude d'activités langagières orales à l'école enfantine genevoise (Thèse de doctorat en Sciences de l'éducation, Université de Genève).

Vanhulle, Sabine. (2009). Des savoirs en jeu en savoir en je. Cheminements réflexifs et subjectivation des savoirs chez des jeunes enseignants en formation. Berne/Neuchâtel : Peter Lang.

Véronique, Daniel (dir.). (2009). L'acquisition de la grammaire du français langue étrangère. Paris : Didier.

VinCENT, Guy. (1994). Forme scolaire et modèle républicain. L'éducation de la démocratie. Dans G. Vincent, L'éducation prisonnière de la forme scolaire? Scolarisation et socialisation dans les sociétés industrielles (p. 207-227). Lyon : Presses universitaires de Lyon. 